(C) [2006] IEEE. Reprinted, with permission, from [S.W. Su, L. Wang, B.G. Celler and A.V. Savkin, Heart Rate Control During Treadmill Exercise, Engineering in Medicine and Biology Society, 2005. IEEE-EMBS 2005. 27th Annual International Conference of the, 17-18 Jan. 2006]. This material is posted here with permission of the IEEE. Such permission of the IEEE does not in any way imply IEEE endorsement of any of the University of Technology, Sydney's products or services. Internal or personal use of this material is permitted. However, permission to reprint/republish this material for advertising or promotional purposes or for creating new collective works for resale or redistribution must be obtained from the IEEE by writing to pubs-permissions@ieee.org. By choosing to view this document, you agree to all provisions of the copyright laws protecting it 


\title{
Heart Rate Control During Treadmill Exercise
}

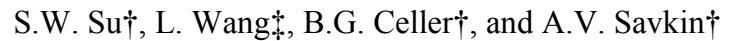 \\ Human Performance Lab, School of Electrical Engineering \& Telecommunications \\ University of New South Wales,UNSW SydneyN.S.W. 2052 Australia \\ Email addresses: †(steven.su, b.celler, a.savkin)@unsw.edu.au, łluwang@student.unsw.edu.au
}

\begin{abstract}
A computer-controlled treadmill and related data collection and processing systems have been developed for the control of heart rate during treadmill exercise. Minimizing deviations of heart rate from a preset profile is achieved by controlling the speed and/or the gradient of the treadmill. A simple and practical heart rate measurement algorithm has been developed to robustly measure the variations of heart rate. Both conventional Proportional-Integral-Derivative (PID) control and fuzzy Proportional-Integral (PI) control approaches have been employed for the controller design. The fuzzy Proportional-Integral algorithm achieved better heart rate tracking performance. Finally, a heart rate based exercising protocol was successfully implemented on the newly designed exercise system.
\end{abstract}

\section{INTRODUCTION}

In sport training, medical diagnosis, rehabilitation and analysis of cardio respiratory kinetics, automated exercise testing systems have revealed their growing importance [1] [2] [3]. These systems can fully implement programmed exercise and training protocols to achieve desired exercising and testing results. The major aim of this study is to develop a computer controlled treadmill system, which can control the heart rate of the subject according to a preset heart rate profile. Some commercial treadmills are already available which offer heart rate control. However, these normally use very simple control strategies, their control performance is poor and they have no mechanism for setting a desired heart rate profile. In this paper we describe a treadmill exercise system which can automatically control the treadmill speed and elevation to accurately track a desired preset heart rate profile.

Conventional PID control is the most popular control algorithms in industry due to its simplicity in structure and ease of tuning. However, an acceptable control performance may be difficult to achieve when the controlled systems are highly nonlinear.

Many physical systems are either highly nonlinear or too complex to control with traditional strategies. However, if an expert can qualitatively describe a control strategy, one can use fuzzy logic to directly translate from the linguistic control rules developed by the expert to a rule base for a fuzzy logic controller. It has been shown that a fuzzy controller can be very effective for controlling a system of unknown structure and nonlinear behavior [4] [5]. In this paper, both conventional PID and fuzzy PI [6] control algorithm have been applied for controller design. Fuzzy PI control resulted in better control performance.

In the next section, we introduce the overall system as well as fuzzy PI control. Section 3 presents heart rate regulation and tracking results achieved. Final conclusions are discussed in Section 4.

\section{SySTEM DESIGN}

The computer controlled treadmill and its related data collection and processing system are shown in Figure 1.

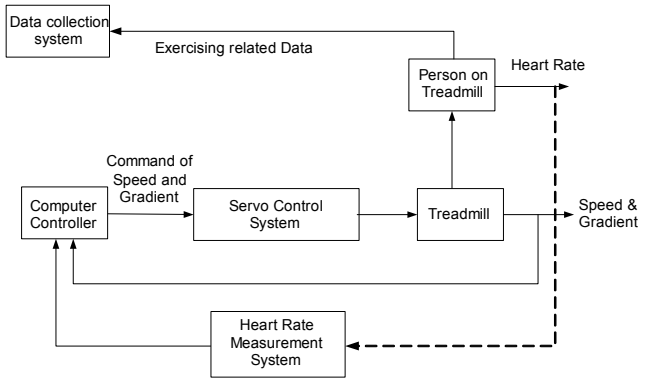

Figure 1. Block diagram for computer controlled treadmill system

The treadmill used in the system is the Powerjog "G" Series fully motorized medical grade treadmill manufactured by Sport Engineering Limited, England. Control of the treadmill can be achieved through an RS232 serial port. The treadmill can receive commands from the computer controller via this link, and obeys such commands without supervision.

There are two main challenges for the control of heart rate. Firstly, the heart rate should be measured with an acceptable accuracy in the presence of external disturbances. Secondly, a suitable control algorithm should be designed to achieve a close match between the exercising hear rate and a desired profile.

\section{A. Heart Rate Measurement}

During exercise and training, we need to collect as much information as possible. Some signals are sensitive to environmental disturbance such as mains frequency noise and electromagnetic interference (EMI) from electrical motors and controllers. This kind of disturbance has been considered during hardware design. 
The measurement of heart rate in the designed control system is implemented using a wireless Polar system. This wireless transmitted signal has been shown to be sensitive to environmental electromagnetic interference. The motor and generator circuit of treadmill produces high frequency (radio frequency) electromagnetic disturbances. The frequency of the generated disturbance is related to the speed of treadmill. It was observed that when the speed of the treadmill is greater than $2.9 \mathrm{~km} / \mathrm{h}$, electromagnetic interference significantly disturbed the polar wireless heart rate measurement system. To overcome this problem, a simple RC EMI filter was applied to directly suppress high frequency noise at the input.

Even in the absence of external interference the heart rate can vary substantially over time under the influence of various internal or external factors [7]. Normally, a moving average filter can be applied to calculate the average (filtered) value and minimize heart rate fluctuations. However, in dynamic control systems, the most current values tend to better reflect the state of the process. A filter that places more emphasis on the most recent data is therefore desirable. An improved exponential weighted moving average filter together with a simple outlier detection algorithm is presented in this paper for the estimation of the heart rate.

Specifically, the control computer collects heart rate signal from a Polar receiver through an analog input (AI) port every 2 seconds, and calculates beat-to-beat interval of heart rate by using an edge detection algorithm.

Assume $\left\{x_{k}\right\}$ is the sequence of the measured interval of heart rate. We apply the exponentially weighted moving average filter to the sequence $\left\{x_{k}\right\}$ :

$\bar{x}_{k}=\alpha \bar{x}_{k-1}+(1-\alpha) x_{k-1}$

The sequence of values $\bar{x}_{k}$ is the filtered version of the original sequence. In the exponentially weighted moving average filter, the filter coefficient $0 \leq \alpha \leq 1$ dictates the degree of filtering. When $\alpha \rightarrow 1$ and a large number of points are being considered, $\bar{x}_{k} \rightarrow \bar{x}_{k-1}$. This means that the most recent measurement does not play a part in the calculation of the average. With the decreasing $\alpha$, the filter places more importance on more recent data by discounting older data in an exponential manner. On the other extreme, if $\alpha \rightarrow 0, \bar{x}_{k} \rightarrow x_{k}$. This means that virtually no filtering is being performed. In this study, the value of $\alpha$ was selected as 0.35 , to achieve the best filtering effect.

Only measured intervals within a reasonable range (for example, between 0.3 and 1.1 seconds) were counted in the measured sequence $\left\{x_{k}\right\}$ in order to remove outliers.

\section{B. Controller Design}

In our computer controlled system, we can select speed and/or gradient of the treadmill as control inputs. It was observed that the heart rate and the speed (or gradient) of the treadmill are related at steady state by a strong nonlinear relationship. To avoid a strong nonlinear relationship between inputs and outputs of the controlled system, the beat-to-beat interval (secs) instead of the heart rate (bpm) was selected as the controlled output. Because the response time of heart rate variation is much longer than 6 seconds, the sampling period of the control system was selected as $T=6$ seconds.

In this study, both PID and fuzzy PI control of heart rate was designed and tested. The block diagram of a typical digital PID or fuzzy PI control system is shown in Figure 2.

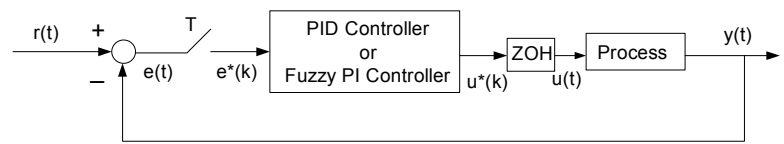

Figure 2. The block diagram of a typical digital PID or fuzzy PI control system

The PID controller is widely used in the industrial application due to its simplicity. The discretized and incremental form of the PID type controller is expressed by

$$
\begin{aligned}
\Delta u^{*}(k)= & u^{*}(k)-u^{*}(k-1)=K_{P_{i}}\left[e^{*}(k)-e^{*}(k-1)\right]+K_{l i} e^{*}(k) \\
& +K_{D i}\left[e^{*}(k)-2 e^{*}(k-1)+e^{*}(k-2)\right]
\end{aligned}
$$

where, $e^{*}(k)=r^{*}(k)-y^{*}(k)$, and $r^{*}(k), y^{*}(k)$ and $e^{*}(k)$ are the reference, output, and error, respectively, at the kth instant. The coefficients $K_{P i}, K_{D i}$, and $K_{I i}$ are the proportional, derivative and integral gains of digital PID controller respectively. These coefficients were tuned using the Ziegler-Nichols method.

In order to improve the control performance of the above PID controller, a simple fuzzy PI control was developed which required no additional hardware. The structure of the fuzzy PI controller is shown in Figure 3.

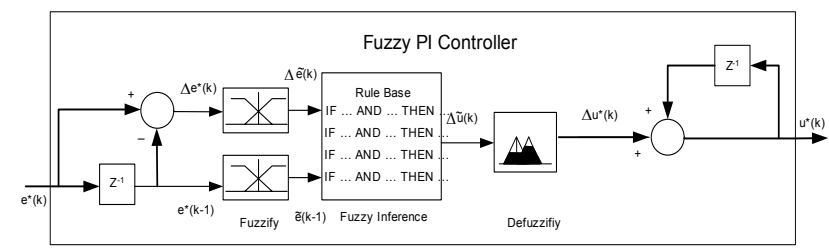

Figure 3. The block diagram of a fuzzy PI controller

In Figure 3, the fuzzy logical control part has two inputs $\Delta e^{*}(k)\left(\Delta e^{*}(k)=e^{*}(k)-e^{*}(k-1)\right)$ and $e^{*}(k-1)$, and an output $\Delta u^{*}(k)$. The fuzzy sets for inputs $\Delta e^{*}(k)$ and $e^{*}(k-1)$ are $\{$ n.de.,p.de. $\}$ and $\{$ n.e.,p.e.\} respectively for outputs $\{n . d u ., z . d u ., p . d u$.$\} .$

Triangular membership functions of both inputs were chosen for the fuzzification module, as shown in Figure 4. 


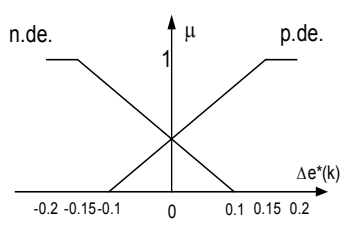

a. $\Delta e^{*}(k)$

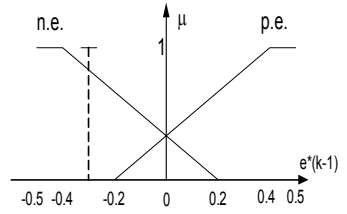

b. $e^{*}(k-1)$
Figure 4. Membership functions of inputs.

The fuzzification module converts the inputs into fuzzy sets, in order to make it compatible with the fuzzy set representations in the rule-antecedent.

There are four rules for fuzzy inference modular in our designed fuzzy controller:

(1) $\operatorname{IF} \Delta e^{*}(k)$ is $n \cdot d e$. AND $e^{*}(k-1)$ is n.e. THEN $\Delta \widetilde{u}(k)$ is $n \cdot d u$,

(2) $\operatorname{IF} \Delta e^{*}(k)$ is $n . d e$. AND $e^{*}(k-1)$ is p.e. THEN $\Delta \widetilde{u}(k)$ is $\mathrm{z} . d u$,

(3) $\operatorname{IF} \Delta e^{*}(k)$ is $p . d e$. AND $e^{*}(k-1)$ is n.e. THEN $\Delta \tilde{u}(k)$ is $z . d u$,

(4) $\operatorname{IF} \Delta e^{*}(k)$ is $p . d e$. AND $e^{*}(k-1)$ is p.e. THEN $\Delta \widetilde{u}(k)$ is $p . d u$.

We apply the MAX-MIN method to implement fuzzy inference. The "center of mass" [8] [9] formula was used to implement defuzzification.

We can use the approach provided in [9] to prove that the above simple fuzzy PI controller is equivalent to a nonlinear PI controller whose proportional and integral gains change with $\Delta e^{*}(k)$ and $e^{*}(k-1)$.

\section{Heart RAte Regulation Results}

The speed of treadmill was initially selected as the control input. The step responses of heart rate control by using PID and fuzzy PI control are shown in Figure 5. It can be seen that fuzzy PI controller achieved better performance than conventional PID control.

The gradient of the treadmill was then used as the control input. The step response (fuzzy PI control) is shown in Figure 6. It can be observed that the heart rate has small fluctuations when the treadmill gradient is selected as the control input. Another potential advantage is that this control mode can be applied to investigate the relationship between the subject's metabolic energy expenditure and their walking pace and heart rate.
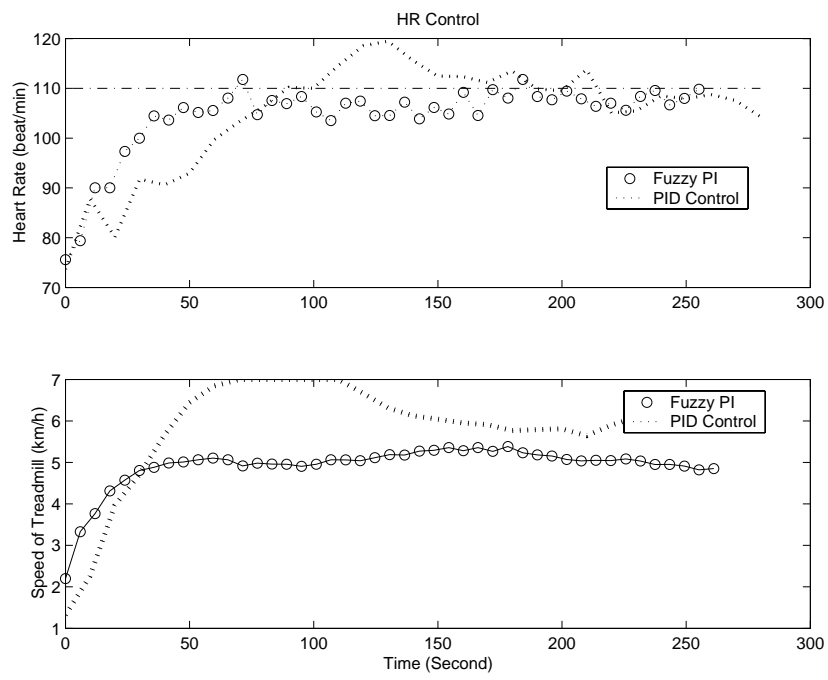

Figure 5. Step change responses of PID and Fuzzy PI controllers.
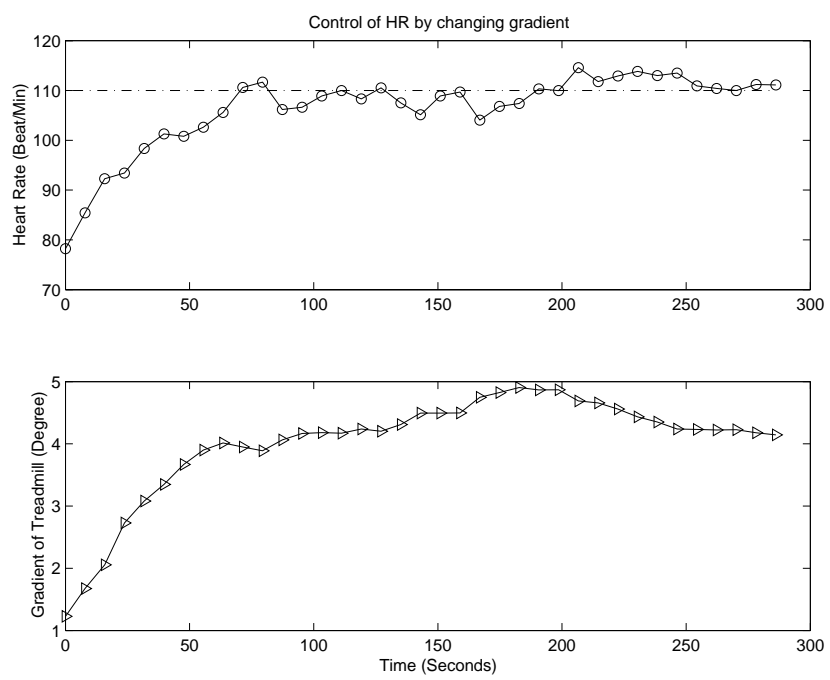

Figure 6. Control of heart rate by changing treadmill gradient.

Finally, a fuzzy PI controller was used to track a preset desired heart rate profile. The profile includes three stages: 5 minutes increasing (from resting heart rate to 110 beats/min), 15 minutes holding (110 beats $/ \mathrm{min})$, and 5 minutes decreasing (from 110 beats/min to normal.). This is comparable to heart rate profiles recommended for optimal aerobic training at about $60 \%$ of maximum effort. Figure 7 below demonstrates that quite satisfactory tracking of the desired heart rate profile was achieved. 

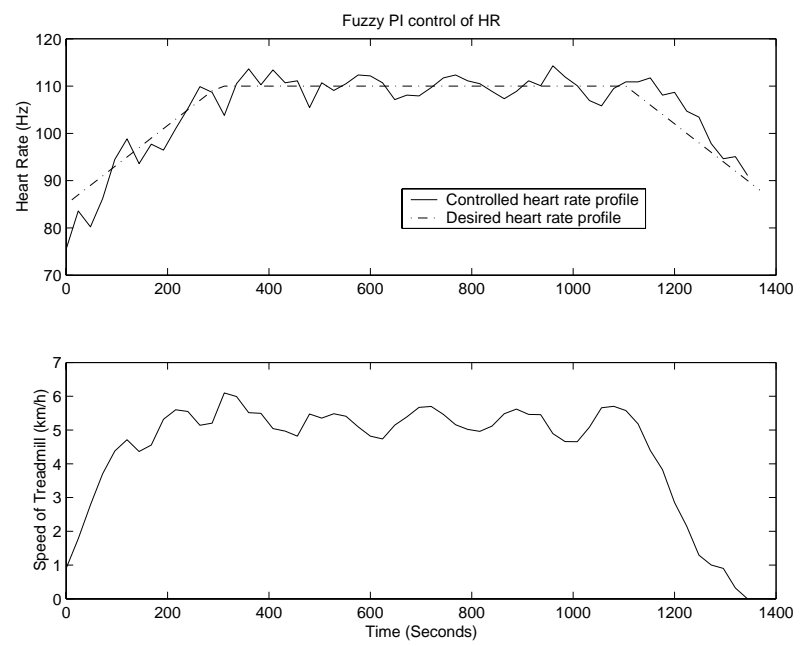

Figure 7. Heart rate tracking for an exercising heart rate profile.

\section{CONCLUSION}

In this study, an automated heart rate regulation system for exercise on a motorized treadmill has been developed. Good heart rate tracking results were achieved using a computer control based on fuzzy logic control. We believe that the ability to track a predetermined heart rate profile may be useful in cardiac rehabilitation programs or for safer exercise for individuals at risk. In the next step, model based control approaches will be investigated to further enhance the performance of heart rate tracking.

\section{ACKNOWLEDGMENT}

The authors gratefully acknowledge the financial support of the Australian Research Council (Grant DP0452186).

\section{REFERENCES}

[1] H. K. A. Lakomy, I. Cambell, and C. Williams, Treadmill performance and selected physiological characteristics of wheelchair athletes, British J. Sports Medicine, vol. 21, no. 3, pp. 130-133, 1987. Rehabilitation Engineering Applied to Mobility and Manipulation.

[2] R. A. Cooper, S. M. Horvath, J. F. Bedi, D. M. Drechsler-Parks, and R. E. Williams, Maximal exercise responses of paraplegic wheelchair road racers, Paraplegia, vol. 30, pp. 573-581, 1992.

[3] Cooper RA, Fletcher-Shaw TL, and Robertson RN, Model Reference Adaptive Control of Heart Rate During Wheelchair Ergometry, IEEE Transactions on Control Systems Technology, Vol. 6, No. 4, pp. 507-514, 1998.

[4] H.R. Berenji, Fuzzy Logic Controllers, in: R.R Yager, L.A. Zadeh (Eds.), An Introduction to Fuzzy Logic Appication in Intelligent Systems, Kluwer, Boston, MA, 1992.
[5] H.J. Zimmermann, Fuzzy Sets Theory and its Applications, Kluwer, NijhoA, Boston, Dordrecht, Lancaster, 1984.

[6] Patel AV and Mohan B. M., "Analytical structures and analysis of the simplest fuzzy PI controllers," Automatica, vol. 38, pp. 981 - 993, June 2002.

[7] G.B. Stanley, K. Poolla, R.A. Siegel, Threshold modeling of autonomic control of heart rate variability. IEEE Trans Biomed Eng 47: 1147-1153, 2000.

[8] H.A. Malki, H.D. Li, G. Chen, New design and stability analysis of fuzzy prepositional-derivative control systems, IEEE Trans.Fuzzy Systems 2 (1994) 245-254.

[9] H. Ying, W. Siler, J.J. Buckeley, Fuzzy control theory a nonlinear case, Automatica 26 (1990) 513-520. 